\title{
Jours fériés et fêtes religieuses
}

Anne Fornerod

\section{OpenEdition}

\section{Journals}

Édition électronique

URL : http://journals.openedition.org/rdr/439

DOI $: 10.4000 /$ rdr.439

ISSN : 2534-7462

\section{Éditeur}

Presses universitaires de Strasbourg

\section{Édition imprimée}

Date de publication : 14 novembre 2019

Pagination : 175-187

ISBN : 979-10-344-0054-6

ISSN : 2493-8637

\section{Référence électronique}

Anne Fornerod, « Jours fériés et fêtes religieuses », Revue du droit des religions [En ligne], 8| 2019, mis en ligne le 25 novembre 2019, consulté le 19 novembre 2020. URL : http://journals.openedition.org/ rdr/439; DOI : https://doi.org/10.4000/rdr.439

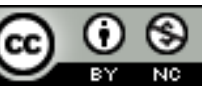

La revue du droit des religions est mise à disposition selon les termes de la Creative Commons Attribution - Pas d'Utilisation Commerciale 4.0 International - CC BY-NC 4.0. 


\section{JOURS FÉRIÉSETFÊTES RELIGIEUSES}

\section{Anne FORNEROD ${ }^{1}$}

Université de Strasbourg / CNRS, Droit, Religion, Entreprise et Société (DRES)

\section{RÉsUMÉ}

Historiquement, les jours fériés reconnus par la loi correspondent pour un certain nombre d'entre eux à des fêtes religieuses chrétiennes. Sous l'effet de la sécularisation, ces jours ont perdu une grande partie et pour certains la totalité de leur lien avec une pratique cultuelle. Or, la pluralisation des courants religieux se traduit par des demandes qui renvoient au contraire à un lien étroit entre fêtes et pratiques religieuses. C'est ce qu'illustre la question des autorisations d'absence pour fêtes religieuses tant pour les agents publics que les employés du secteur privé.

\section{ABstract}

Historically, the law enshrines public holidays that correspond to Christian feasts. As a result of secularization, these days have lost a large part and for some of them their entire link with religious practice. However, at the same time, the pluralization of religious currents is reflected in requests that refer to a close link between holidays and religious practices. This is illustrated by the issue of leave of absence for religious holidays for both public and private sector employees.

1. Une version en allemand de cet article est publiée dans le ${ }^{\circ}$ (66) 2019/1 de österreichisches Archiv für recht \& religion. 
$\mathrm{L}^{2}$ e 22 janvier 2019, dans l'arrêt Cresco Investigation GmbH c. Markus Achatzi, la Cour de justice de l'Union européenne s'est prononcée sur la législation autrichienne sur le repos et les jours fériés qui prévoit que le Vendredi saint est, sous certaines conditions, un jour férié chômé. En effet, ce jour est férié et chômé uniquement pour les salariés membres de quatre Églises chrétiennes et la loi leur permet de choisir entre bénéficier de ce jour de congé supplémentaire ou travailler et recevoir alors une indemnité qui s'ajoute à leur salaire. Or, selon la Cour de Luxembourg,

«l'article $1^{\text {er }}$ et l'article 2, paragraphe 2, de la directive 2000/78 doivent être interprétés en ce sens qu'une législation nationale en vertu de laquelle, d'une part, le Vendredi saint n'est un jour férié que pour les travailleurs qui sont membres de certaines églises chrétiennes et, d'autre part, seuls ces travailleurs ont droit, s'ils sont amenés à travailler durant ce jour férié, à une indemnité de jour férié constitue une discrimination directe en raison de la religion ${ }^{2} »(\S 69)$.

Une telle situation serait-elle envisageable en droit français? Il convient d'emblée de souligner l'absence d'une législation qui pourrait donner lieu à une solution comparable. Pour autant, les fêtes religieuses ont, ces dernières années, suscité des discussions relatives au principe d'égalité entre agents publics, faisant ainsi écho à l'affaire autrichienne, tout en illustrant la primauté du principe d'égalité sur celui de non-discrimination. Plus largement, en droit français, la question du statut des fêtes religieuses est complexe dans la mesure où elle concentre différentes façons de les appréhender. La terminologie reflète cette complexité: les expressions de jours fériés, jours chômés, fêtes religieuses, fêtes légales ne pouvant être tenues pour équivalentes ou ne coïncidant qu'imparfaitement ${ }^{3}$. Une approche historique est incontournable, dans la mesure où ce statut connaît continuellement des évolutions, juridiquement, mais aussi sociologiquement. En outre, c'est non seulement le droit du travail qui est pertinent en la matière, mais également les différents régimes locaux et le droit des religions en ce qu'il reflète les adaptations des normes aux évolutions des pratiques religieuses. En effet, ce statut paraît mouvant principalement sous les effets conjugués de la sécularisation des pratiques religieuses chrétiennes à l'origine de la majorité des jours fériés, de la diversification des croyances religieuses et donc des fêtes ainsi que des évolutions du droit du travail qui fixe les jours fériés. La réglementation des fêtes légales partage avec la règle du repos dominical l'influence d'un

2. CJUE, 22 janv. 2019, C -193/17, Cresco Investigation GmbH c. Markus Achatzi.

3. Sur ce point, V. J. Lalouette, Jours de fête. Jours fériés et fêtes légales dans la France contemporaine, Paris, Tallandier, 2010, p. 11-12. 
contexte sociologique et politique sur leur lien originel avec une doctrine religieuse qui a marqué pendant des siècles le rythme des activités professionnelles ${ }^{4}$. En reliant les différentes facettes des fêtes religieuses dans leurs évolutions, il est possible de distinguer entre deux régimes principaux, à savoir celui des fêtes religieuses qui sont devenues fêtes légales - ou jours fériés - (1) et celui de celles qui conservent leur statut de pratique religieuse stricto sensu et sont régies comme telle. En effet, autant la sécularisation a contribué à associer fêtes religieuses et jours fériés, autant la pluralisation des convictions religieuses au-delà du christianisme a conduit à maintenir un lien entre fête religieuse et liberté de pratiquer sa religion (2). Surtout, se pose la question du sens, en droit, de maintenir légalement le caractère férié de fêtes du calendrier chrétien dans un contexte aujourd'hui connu de différenciation profonde entre les pratiques correspondant à la stricte observance de prescriptions religieuses et ce qui relève bien plus de coutumes et d'usages procédant d'un héritage historique et culturel. Aussi le débat sur une évolution du statut des fêtes religieuses est-il loin d'être clos (3).

\section{LES FÊTES RELIGIEUSES, JOURS FÉRIÉS LÉGAUX}

L'articulation entre les fêtes religieuses et les jours fériés consacrés par la législation civile a véritablement commencé au lendemain de la Révolution, période de rupture pendant laquelle «l'objectif explicite de supplanter les fêtes chrétiennes avait clairement échoué ${ }^{5} »$. L'article 41 du Concordat de 1801 traduit la mainmise de la législation civile sur le calendrier religieux dans la mesure où il dispose qu'«Aucune fête, à l'exception du dimanche, ne pourra être établie sans la permission du Gouvernement». Dès 1802, l'arrêté du 29 germinal an X (19 avril 1802) prévoit que «les jours de fêtes qui seront célébrés en France, outre les dimanches, sont: La Naissance de Notre-Seigneur Jésus-Christ, l'Ascension, l'Assomption de la très-sainte Vierge, la fête de tous les Saints ${ }^{6}{ }$. Si la reconnaissance officielle de quatre

4. Ainsi, alors que la règle du repos dominical « fut constante pendant tout l'Ancien Régime », par la suite, "les radicaux de la $\mathrm{III}^{\mathrm{e}}$ République n'estimaient pas souhaitable d'établir un jour de repos obligatoire pour tous, considérant que cette règle était contraire tant à la liberté du travail qu'à la laïcité»: S. Hennion-MoreAu, «La règle du repos dominical», Droit social 1990, p. 434.

5. A. Join-Lambert, «Quel sens pour les fêtes chrétiennes?», Études, n 3, 2010, p. 356.

6. Cité par H.-F. Rivière, Codes français et lois usuelles, Paris, A. Marescq Ainé, $4^{e}$ éd. 1878, p. 12. Pour les catholiques, les fêtes religieuses sont les fêtes de précepte (ou d'obligation) que prescrit le Code de droit canonique (can. 1246 \& 1). Toutefois, les fidèles suivent la liste déterminée par l'Église locale (la Conférence des évêques). 
fêtes religieuses tranche avec le «laïcisme» révolutionnaire, il serait erroné d'y voir «une affirmation triomphante du retour du catholicisme» dans la mesure où le nombre des fêtes d'obligation est nettement réduit et vaine la perspective d'un retour à l'Ancien Régime, où «les jours chômés se comptaient encore par dizaines durant les $\mathrm{XVII}^{\mathrm{e}}$ et $\mathrm{XVIII}^{\mathrm{e}}$ siècles ${ }^{7} »$.

À ces quatre jours établis en 1802 s'ajouteront les lundis de Pâques et de Pentecôte en vertu de la loi du 18 mars 1886. La loi de séparation des Églises et de l'État du 9 décembre 1905 n'apportera pas de modification à cette liste ${ }^{8}$.

Aujourd'hui, en vertu de l'article L. 3133-1 du Code du travail, la liste des fêtes légales constituant des jours fériés est la suivante: " $1^{\circ}$ Le $1^{\text {er }}$ janvier; $2^{\circ}$ Le lundi de Pâques; $3^{\circ}$ Le $1^{\text {er }}$ mai; $4^{\circ}$ Le 8 mai; $5^{\circ}$ L'Ascension; $6^{\circ}$ Le lundi de Pentecôte; $7^{\circ}$ Le 14 juillet; $8^{\circ}$ L'Assomption; $9^{\circ}$ La Toussaint; $10^{\circ}$ Le 11 novembre; $11^{\circ}$ Le jour de Noël.» Le Code du travail précise à propos du seul $1^{\text {er }}$ mai qu'il est "jour férié et chômé» (art. L. 3133-4), remettant le sort des autres fêtes légales à «un accord d'entreprise ou d'établissement ou, à défaut, une convention ou un accord de branche» (art. L. 3133-3-1).

$\mathrm{Au}$ titre des dispositions particulières applicables dans les départements de la Moselle, du Bas-Rhin et du Haut-Rhin, le Code du travail ajoute deux jours fériés à la liste établie en droit général (art. L. 3134-13) : «le Vendredi Saint dans les communes ayant un temple protestant ou une église mixte» et le «second jour de Noël» et, surtout, il précise que les - au total treize jours fériés sont chômés ${ }^{9}$. Enfin, il est à noter qu'en outre-mer (sont concernés la Guadeloupe, la Guyane, la Martinique, Mayotte ${ }^{10}$, La Réunion, Saint-Barthélemy et Saint-Martin), le jour de commémoration de l'abolition de l'esclavage est un jour férié (C. trav., art. L. 3422-2).

Sur les onze jours que comprend la liste de droit commun, l'on s'aperçoit très rapidement que plus de la moitié correspondent à des fêtes religieuses chrétiennes ou revêtent une connotation religieuse - toujours chrétienne. La

7. J. Lalouette, op.cit., p. 21-22.

8. L'article 42 de la loi de 1905 disposait que «Les dispositions légales relatives aux jours actuellement fériés sont maintenues». Cet article fut abrogé par la loi $n^{\circ}$ 73-4 du 2 janvier 1973 relative au Code du travail dans lequel devait alors figurer la liste des jours fériés.

9. V. L. Grisey Martinez, "Jours fériés», in Le guide du droit local. Le droit applicable en Alsace et en Moselle de A à Z, Strasbourg, Institut du droit local alsacien-mosellan, 2015, p. 257-258.

10. Sur le cas particulier de Mayotte dont la population est en majorité de religion musulmane, V. C. Viard, «Congés... pour "prières" », Droit et cultures, n 51, 2006, p. 62. 
principale modification apportée à cette liste, particulièrement stable pour ce qui concerne les fêtes à connotation religieuse, fut le changement de statut - provisoire ${ }^{11}$ - du lundi de Pentecôte en 2004. Le contexte est celui de la loi $n^{\circ}$ 2004-626 du 30 juin 2004 relative à la solidarité pour l'autonomie des personnes âgées et des personnes handicapées adoptée en réaction au décès de plusieurs milliers de personnes au cours de l'été 2003 en raison de la canicule. Le financement de cette solidarité prend alors la forme d'une journée supplémentaire de travail non rémunéré, dont le salaire correspondant doit être reversé à un fonds de solidarité en faveur de l'autonomie des personnes âgées ou handicapées. Le lundi de Pentecôte avait été retenu pour cette journée de solidarité, sauf convention, accord de branche ou accord d'entreprise décidant d'un autre jour précédemment non travaillé.

Un rapport parlementaire préalable à la loi nous renseigne sur le choix du lundi de Pentecôte. Ce choix découlerait d'une part «de son statut de jour férié légal et de l'intérêt de prévoir le travail d'un jour relevant de cette catégorie, parce que chômé par la très grande majorité des Français ${ }^{12}$ ». D'autre part, «si le choix du lundi de Pentecôte n'est pas exempt d'inconvénients pour certaines manifestations religieuses, mais également culturelles ou sportives, il apparaît cependant comme le choix le plus raisonnable et celui heurtant le moins la mémoire collective et les traditions culturelles de notre pays». L'on apprend surtout qu'il a été procédé selon une «logique d'élimination» parmi la liste des jours fériés ${ }^{13}$.

11. En effet, par la suite, la loi no 2008-351 du 16 avril 2008 a maintenu la journée de solidarité, mais en supprimant toute référence au lundi de Pentecôte.

12. France. Assemblée nationale, Rapport fait au nom de la commission des affaires culturelles, familiales et sociales sur le projet de loi $\left(n^{\circ} 1350\right)$ relatif à la solidarité pour l'autonomie des personnes âgées et des personnes handicapées, n 1540, 2004, p. 45.

13. Ibid., p. 46. Ainsi «- le $1^{\text {er }}$ janvier est un jour légalement férié depuis un avis du Conseil d'État du 23 mars 1810 et aucune voix ne semble s'être élevée pour proposer d'en faire la journée de solidarité;

- de même, nul ne saurait songer au $1^{\text {er }}$ mai au statut réellement à part;

- le lundi de Pâques (légalement férié depuis 1886), l'Ascension, le 15 août et le 25 décembre (fériés depuis un arrêté du 29 germinal an X) constituent des fêtes religieuses majeures profondément ancrées dans la vie culturelle française;

- le 14 juillet (férié depuis une loi du 6 juillet 1880) constitue quant à lui une fête laïque tout aussi profondément ancrée;

- plus récents, le 8 mai (un temps remis en cause sous le septennat de M. Valéry Giscard d'Estaing et rétabli par une loi du 2 octobre 1981) et le 11 novembre (en vertu de la loi du 24 octobre 1922) constituent cependant des dates profondément symboliques, commémorant la fin de conflits qu'il convient de garder en mémoire; ils posent la question récurrente de l'opportunité de créer un Memorial Day à la française, un jour du Souvenir, et se heurtent à l'obstacle tout aussi récurrent du choix d'une date pour un tel jour qui rencontrerait à l'évidence - au vu des tensions que provoque le lundi de Pentecôte - des oppositions farouches quelle que soit la date retenue; 
La mise en œuvre de la législation relative à la journée de solidarité a donné lieu à contentieux, entre autres sur son champ d'application. L'on retiendra plus particulièrement un arrêt de la cour administrative d'appel de Bordeaux du $1^{\text {er }}$ avril $2008{ }^{14}$ qui présente l'intérêt de distinguer entre le régime de cette journée et celui des autorisations d'absence pour fêtes religieuses. En l'espèce, une note du préfet de Mayotte imposait aux agents publics de la collectivité départementale de Mayotte de travailler le 16 mai 2005, lundi de Pentecôte. Le requérant mettait en avant que les agents publics de confession musulmane avaient pu demander des autorisations d'absence pour l'Aïd el Kebir et Mawlid Annabawi - nous sommes dans le contexte social et religieux spécifique de Mayotte -, mais que «les agents publics de l'État de confession catholique n'ont pu bénéficier de telles autorisations pour le lundi de la Pentecôte, ce qui constitue une rupture d'égalité de traitement». Or, il a été jugé que la note du préfet «n'a ni pour objet ni pour effet de fixer le régime des autorisations d'absence dont peuvent bénéficier les agents publics de confession musulmane pour assister à des fêtes religieuses; qu'il suit de là que le moyen tiré de la rupture d'égalité entre les agents publics de confession catholique et ceux de confession musulmane ne peut être utilement invoqué». En d'autres termes, il convient de distinguer entre les jours fériés établis par le Code du travail et les autorisations d'absence pour fêtes religieuses, dont la liste est expressément et clairement établie pour les agents publics. Dans ce cas en effet, les autorisations d'absence procèdent d'une philosophie différente, à savoir l'exercice de la liberté de religion.

\section{LES FÊTES RELIGIEUSES, PRATIQUES CONVICTIONNELLES}

En dehors de la consécration par la loi qui les transforme en fêtes légales, les fêtes religieuses peuvent être des jours chômés. L'on pourrait imaginer a priori que le fondement juridique de ce chômage change pour passer du droit du travail à l'exercice de la liberté de religion de l'individu qui se réclame de ses convictions religieuses pour demander une autorisation d'absence professionnelle. De ce point de vue, seule la Déclaration pour l'élimination de toutes les formes d'intolérance et de discrimination sur la base de la religion et de la croyance, adoptée en 1981 par l'Assemblée générale de l'ONU,

- bon nombre de ces fêtes ne peuvent en outre être retenues du fait qu'elles tombent certaines années sur des jours ordinairement non travaillés par la majorité des Français, le samedi ou le dimanche.»

14. CAA Bordeaux, $1^{\text {er }}$ avr. 2008, nº 06BX01003. 
inclut expressément dans le champ d'application de la liberté de religion «la liberté d'observer les jours de repos et de célébrer les fêtes et cérémonies conformément aux préceptes de sa religion ou de sa conviction» (art. 6). En droit français, la possibilité de s'absenter pour prendre part à une fête religieuse est réglementée diversement selon que l'employé est un agent public ou travaille dans le secteur privé.

Les demandes d'absence des agents publics, régies de longue date dans la fonction publique, relève de l'équilibre entre leur obligation de neutralité et le respect de leur liberté de religion.

Ainsi, la loi no 83-634 du 13 juillet 1983 portant droits et obligations des fonctionnaires (telle que modifiée par la loi n 2016-483 du 20 avril 2016 relative à la déontologie et aux droits et obligations des fonctionnaires) dispose que «le fonctionnaire exerce ses fonctions avec dignité, impartialité, intégrité et probité. Dans l'exercice de ses fonctions, il est tenu à l'obligation de neutralité. Le fonctionnaire exerce ses fonctions dans le respect du principe de laïcité. À ce titre, il s'abstient notamment de manifester, dans l'exercice de ses fonctions, ses opinions religieuses» (art. 25). Toutefois, la même loi prévoit que «la liberté d'opinion est garantie aux fonctionnaires» et qu' «aucune distinction, directe ou indirecte, ne peut être faite entre les fonctionnaires en raison de leurs opinions politiques, syndicales, philosophiques ou religieuses [...]» (art. 6). Le lien entre une autorisation d'absence et l'exercice de la liberté de religion n'a été explicitement établi qu'au sujet d'une absence régulière, dans une ordonnance du Conseil d'État du 16 février $2004^{15}$. Concernant une fête religieuse à proprement parler, c'est un arrêt plus ancien du Conseil d'État qui a fixé la règle selon laquelle «le régime des autorisations d'absence des fonctionnaires constitue au même titre que les congés proprement dits un élément du statut des intéressés». En l'espèce, la requérante, agent non titulaire et hôtesse d'accueil au Centre Georges Pompidou, s'était vu refuser des autorisations d'absence pour le Vendredi saint, la fête Dieu et la fête de la médaille miraculeuse. Or, la Haute juridiction a considéré «qu'en l'absence de règles définissant le régime des autorisations d'absence, le directeur du centre précité a rejeté la demande

15. V. CE, ord., 16 févr. 2004, no 264314, Benaissa: il s'agissait d'un agent d'entretien et gardien d'immeuble de l'office municipal d'habitations à loyer modéré de Saint-Dizier auquel avait été refusée l'autorisation de s'absenter une heure tous les vendredis en début d'après-midi pour aller à la mosquée. Le Conseil d'État a consacré à cette occasion la liberté de culte comme une liberté fondamentale, mais a jugé en l'espèce que «les nécessités $\mathrm{du}$ fonctionnement normal du service public faisaient obstacle à ce que $\mathrm{M}$. Benaissa soit autorisé à se rendre à la mosquée chaque vendredi de 14 heures à 15 heures». 
en se fondant par sa décision du 10 mars 1987, sur ce que "Seules... les fêtes religieuses légales en France peuvent donner lieu à autorisation d'absence" », alors que "l'institution par la loi de fêtes légales ne [faisant] pas, par elle-même, obstacle à ce que, sous réserve des nécessités du fonctionnement normal du service, des autorisations soient accordées à des agents publics pour participer à d'autres fêtes religieuses correspondant à leur confession, le directeur du centre national d'art et de culture Georges Pompidou a entaché sa décision d'erreur de droit ${ }^{16} »$. Le Conseil d'État a par la suite considéré à propos de la circulaire du 10 décembre 2010 sur les règles de vie quotidienne dans les services déconcentrés de la direction générale des Finances publiques (temps de travail et autorisation d'absence) qui prévoyait entre autres que les autorisations d'absence pour des fêtes religieuses « concernent les seules religions pour lesquelles la fonction publique assure une publication annuelle», "qu'il appartient toutefois au chef de service d'apprécier au cas par cas si l'octroi d'une autorisation d'absence sollicitée par un agent pour participer à une fête autre que l'une des fêtes religieuses légales est compatible avec les nécessités du fonctionnement normal du service» et «qu'en interdisant, en dehors des fêtes légales, l'octroi d'autorisation pour des fêtes religieuses autres que celles dont la direction générale de l'administration et de la fonction publique publie annuellement la liste, au demeurant indicative, le ministre a entaché la circulaire d'excès de pouvoir ${ }^{17}$ ».

Certes, «les autorisations d'absence pour motif religieux ne constituent pas un droit pour les agents publics », mais elles doivent faire l'objet d'un examen au cas par cas. Une interdiction générale d'accorder de telles autorisations adoptée par un chef de service serait «en contradiction avec la volonté du législateur et l'esprit des circulaires fixant annuellement une liste indicative des principales fêtes religieuses des différentes confessions ${ }^{18}$ ».

Le juge administratif s'inscrit clairement, tout en le précisant, dans l'esprit de la législation applicable qui tient dans une circulaire, régulièrement reprise, du 23 septembre 1967 relative aux autorisations d'absence pour fêtes religieuses ${ }^{19}$. Elle concerne «les fonctionnaires désireux de participer à des

16. CE, 12 févr. 1997, n 125893, Mademoiselle Henny.

17. CE, 26 oct. 2012, n $\mathrm{n}^{\circ} 346648$, Lliboutry.

18. Défenseur des droits, déc. no 2014-061, 29 juill. 2014. En l'espèce, le réclamant s'était vu refuser par les services de la mairie où il travaillait, deux années consécutives, une autorisation d'absence de trois jours pour célébrer les fêtes du Yom Kippour et de Rosh Hashana: https://juridique.defenseurdesdroits.fr/doc_num.php?explnum_id=10732 [consulté le 27 mai 2019].

19. Circ. n FP/901, 23 sept. 1967, relative aux autorisations d'absence pour fêtes religieuses. 
fêtes ou à des cérémonies religieuses qui ne sont pas inscrites au calendrier des fêtes chômées tel que celui-ci est fixé par la législation et par l'usage». La circulaire devenue pérenne du 10 février 2012 relative aux autorisations d'absence accordées à l'occasion des principales fêtes religieuses des différentes confessions rappelle que les chefs de service «peuvent accorder à leurs agents une autorisation pour participer à une fête religieuse correspondant à leur confession dans la mesure où cette absence est compatible avec le fonctionnement normal du service» et, à la différence de la circulaire de 1967, assortit ce rappel d'une liste de fêtes ${ }^{20}$.

Dans le secteur professionnel privé, le Code du travail ne régit pas expressément la question des absences pour fêtes religieuses. Pour autant, une règle équivalente vient à s'appliquer, qui vise à respecter la compatibilité entre l'absence pour fête religieuse et la bonne marche de l'entreprise. L'employeur peut déterminer en amont les règles en la matière qui peuvent consister en des aménagements d'horaires par exemple ou autoriser au cas par cas une telle absence, mais qui sera décomptée des jours de congés du salariée ${ }^{21}$. À titre d'exemple, un salarié a pu être licencié pour faute grave du fait, notamment, qu'il avait, un 31 mars, "participé toute la journée à une fête religieuse en l'honneur du dieu Shiva». Or, «la demande de congés déposée pour cette journée par Monsieur S. avait été refusée par son employeur et, d'autre part, [...] le 31 mars ne constitue pas un jour férié $22 »$. Par ailleurs, le fait que l'employé prenne des congés sans solde est sans incidence sur la nécessité pour lui d'obtenir l'autorisation de son employeur qui conserve «en toute hypothèse son pouvoir de direction et d'organisation ${ }^{23} »$.

20. Circ. NOR/MFPF1202144C, 10 févr. 2012. La liste des principales fêtes religieuses est la suivante: les principales fêtes catholiques et protestantes «sont prises en compte au titre du calendrier des fêtes légales». Fêtes orthodoxes: Téophanie: selon le calendrier grégorien ou selon le calendrier julien; Grand Vendredi Saint; Ascension. Fêtes arméniennes: fête de la Nativité; fête des Saints Vartanants; commémoration du 24 avril. Fêtes musulmanes: Aid El Adha; Al Mawlid Ennabi; Aid El Fitr. Fêtes juives: Chavouot (Pentecôte); Roch Hachana (jour de l'an: deux jours); Yom Kippour (Grand pardon). Fête bouddhiste: Fête du Vesak («jour du Bouddha»).

21. Pour plus de détails, V. C. VIARD, art. cit.

22. CA Grenoble, 22 juin 2017, $\mathrm{n}^{\circ}$ 14/04291.

23. CA Paris, 7 déc. 2015, $\mathrm{n}^{\circ}$ 15/04881. 


\section{LES FÊTES RELIGIEUSES : JOURS FÉRIÉS, PRATIQUES CONVICTIONNELLES OU JOURS DE CONGÉS?}

Historiquement, les jours fériés ont été institués non pas pour procurer un temps de repos supplémentaire aux travailleurs, mais pour répondre « d'abord et essentiellement à une autre fonction, religieuse ou politique. Il s'agissait d'organiser la vie en société de telle sorte que les Français pussent suivre les préceptes de l'Église en sanctifiant les fêtes d'obligation, et communier dans l'hommage rendu au souverain, dans l'amour de la Patrie, dans l'adhésion à la République, dans la conviction de former une même nation ${ }^{24} »$.

Encore aujourd'hui, même si la question du chômage des jours fériés correspondant à des fêtes religieuses se pose largement sous l'angle du droit du travail, leur signification sociale et plus largement symbolique demeure un sujet de réflexion. Deux exemples illustrent ce double questionnement.

En premier lieu, la portée symbolique des jours fériés est revenue au premier plan à propos des fêtes des religions non catholiques, dans une société certes sécularisée, mais également plurielle sur le plan religieux. Dans son rapport du 11 décembre 2003, la Commission de réflexion sur l'application du principe de laïcité dans la République (dite Commission Stasi) avait inclus dans sa liste de recommandations de «Faire des fêtes religieuses de Kippour et de l'Aï-El-Kebir des jours fériés dans toutes les écoles de la République. Dans le monde de l'entreprise, permettre aux salariés de choisir un jour de fête religieuse sur leur crédit de jours fériés». Le rapport précise qu'il s'agit de "prendre en considération les fêtes les plus solennelles des religions les plus représentées » sans «remettre en cause le calendrier conçu principalement autour des fêtes catholiques» dans l'objectif de "prendre en considération que le paysage spirituel français a changé en un siècle ${ }^{25} »$. Il est à souligner que le rapport marque une différence entre l'école où les élèves «ne travailleraient pas les jours de Kippour et de l'Aïd-el-Kébir» et le monde de l'entreprise où «le Kippour, l'Aïd-el-Kébir, le Noël orthodoxe ou des chrétiens orientaux seraient reconnus comme jours fériés» et donc pas nécessairement chômés. Dans cette dernière hypothèse, ces jours «seraient substituables à un autre jour férié à la discrétion du salarié. Cette proposition

24. J. Lalouette, op.cit., p. 18.

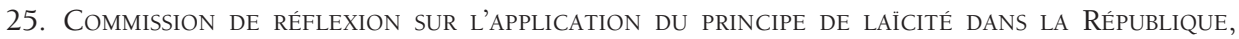
Rapport au président de la République, 2003, p. 65: https://www.ladocumentationfrancaise. fr/var/storage/rapports-publics/034000725.pdf [consulté le 27 mai 2019]. 
serait définie après concertation avec les partenaires sociaux, et en tenant compte des spécificités des petites et moyennes entreprises ${ }^{26}{ }{ }$.

Dès son discours de réception du rapport (17 décembre 2003), le président de la République a fait part de son avis défavorable à cette recommandation. Il estime d'une part que le calendrier scolaire compte déjà beaucoup de jours fériés, tout en précisant que «comme c'est déjà largement l'usage [il] souhaite qu'aucun élève n'ait à s'excuser d'une absence justifiée par une grande fête religieuse comme le Kippour ou l'Ait-El-Kebir, à condition que l'établissement en ait été préalablement informé» et qu'il "va de soi aussi que des épreuves importantes ou des examens ne doivent pas être organisés ces jours-là ${ }^{27} »$. D'autre part, quant au monde de l'entreprise, il n'est pas évoqué sous cet angle des jours fériés, mais sous celui de la réglementation du port de signes religieux.

La question des fêtes religieuses autres que catholiques fut à nouveau soulevée dans le cadre du processus législatif devant conduire à l'adoption de la loi $\mathrm{n}^{\circ}$ 2010-1192 du 11 octobre 2010 interdisant la dissimulation du visage dans l'espace public. Jugeant que «l'une des propositions les plus novatrices du rapport Stasi résidait dans la volonté de prendre en considération les fêtes les plus solennelles des grandes religions présentes sur le territoire national», la mission parlementaire d'information sur la pratique du port du voile intégral sur le territoire national a estimé «que cette idée, qui n'a pas connu de suites, mérite qu'on l'étudie à nouveau ${ }^{28} »$. Pour autant, la proposition fait long feu et disparaît rapidement du dossier législatif ${ }^{29}$.

En second lieu, la prise en compte de la pluralité des croyances à travers les fêtes religieuses dans son articulation avec le nombre de jours de congés dans la fonction publique a fait débat au sein du Parlement, renvoyant à la délicate conciliation entre le principe juridique d'égalité et une situation de

26. Ibid.

27. Discours prononcé par M. Jacques Chirac Président de la République relatif au respect du principe de laïcité dans la République, 2003: http://www.jacqueschirac-asso.fr/archiveselysee.fr/elysee/elysee.fr/francais/interventions/discours_et_declarations/2003/decembre/ fi001576.html [consulté le 27 mai 2019].

28. France. Assemblée nationale, Rapport d'information au nom de la mission d'information sur la pratique du port du voile intégral sur le territoire national, $\mathrm{n}^{\circ} 2262,2010, \mathrm{p} .141$. Disponible à: http://www.assemblee-nationale.fr/13/pdf/rap-info/i2262.pdf [consulté le 27 mai 2019].

29. Ainsi, elle n'apparaît plus, entre autres, dans le rapport fait au nom de la commission des lois constitutionnelles, de la législation et de l'administration générale de la République sur le projet de loi ( $\mathrm{n}^{\circ} 2520$ ), interdisant la dissimulation du visage dans l'espace public du député Jean-Paul Garraud, nº 2648, 2010. 
pluralisme religieux. Ce débat découle du constat que «les agents de l'une des cinq confessions reconnues autres que les religions catholique et protes$\operatorname{tante}^{30}$ sont susceptibles de bénéficier de l'octroi de 1 à 3 journées fériées supplémentaires que leurs collègues ${ }^{31} »$. Le sénateur auteur de la question constate que «les principes républicains d'égalité n'avaient que peu à souffrir de cette tolérance qui était en quelque sorte la juste contrepartie de la confusion historique et culturelle du calendrier chrétien avec une grande partie du calendrier des journées fériées de la République. Aujourd'hui, la variété confessionnelle qui caractérise la société française se retrouve bien évidemment dans la fonction publique». En conséquence, il demande au ministre du Travail, de l'Emploi et de la Santé «si ce qui auparavant relevait de mesure d'exception, ne devrait pas désormais faire l'objet d'une révision dans sa gestion, à défaut de quoi la réalité de l'inégalité pourrait devenir à terme criante». Selon un autre parlementaire, la circulaire du 23 septembre 1967 «génère dans les collectivités territoriales beaucoup d'incompréhension et crée un sentiment d'inégalité, alors que les fêtes légales d'origine civile ou religieuse, qui sont devenues aujourd'hui des jours fériés, bénéficient à tous les agents, et ce indifféremment de leur appartenance à un culte. Face à cette situation, il lui demande comment il entend remédier à cette situation en proposant notamment que ces autorisations [d'absence] soient imputées sur les droits à congés payés ${ }^{32} »$.

Cette discussion a donné lieu à une proposition de loi tendant à assurer l'égalité des droits à congé dans la fonction publique en $2015^{33}$. L'exposé des motifs met en balance la liberté de culte et le principe d'égalité, soulevant la question de savoir si l'on n'est "pas ainsi allé trop loin dans la transcription de la liberté de culte dans le droit du travail, entraînant de facto une discrimination envers ceux et celles qui ne bénéficient que des fêtes légales» et affirmant que «cette proposition de loi tend à rétablir un principe d'égalité entre tous les salariés, considérant que la liberté de culte ne peut à elle seule justifier une inégalité de droit social». En conséquence, il est proposé de

30. Il s'agit d'une référence à la circulaire précitée de 2012, qui vise cinq religions: l'orthodoxie, la religion arménienne, le bouddhisme, le judaisme et l'islam.

31. Question écrite $n^{\circ} 19730$ du sénateur UMP Jean-Paul Fournier: JO Sénat, 4 août 2011, p. 2017. Ultérieurement, une question écrite du sénateur UMP Francis Delattre souligne le risque de rupture d'égalité entre «les salariés sans religion, catholiques ou protestants [qui] se sentent lésés sachant que les autres bénéficient des jours fériés français. », Question $\mathrm{n}^{\circ} 22674$ : JO Sénat, $1^{\text {er }}$ mars 2012, p. 564.

32. Rép. min. no 117286: JOAN, 6 sept 2011, p. 9493, É. MOURrut.

33. Prop. de loi Ass. nationale, $\mathrm{n}^{\circ} 2567,2015$, tendant à assurer l'égalité des droits à congé dans la fonction publique. 
modifier la loi du 13 juillet 1983 portant droits et obligations des fonctionnaires «afin que les jours d'absences pour les fêtes religieuses non inscrites au calendrier des fêtes nationales, soient considérés comme des jours de congés et par là même décomptés du nombre de jours de congés accordés par an à tout fonctionnaire d'État, territorial, ou de la fonction publique hospitalière ».

Demeurée sans suite, cette proposition de loi met au jour la tension entre des jours fériés adossés à une tradition chrétienne, mais qui ont perdu de leur teneur religieuse et la possibilité d'absence pour des fêtes qui ont, elles, conservé toute leur portée rituelle. 